Recherches en didactique des langues et des cultures

Les cahiers de l'Acedle

18-1 | 2021

En quoi les langues ont-elles un rôle à jouer dans les sociétés mondialisées au sein d'une Europe fragilisée ?

\title{
Le paradigme plurilingue pour une révolution humaniste
}

\section{Giovanni Agresti}

\section{OpenEdition}

Journals

Édition électronique

URL : https://journals.openedition.org/rdlc/8564

DOI : $10.4000 /$ rdlc. 8564

ISSN : 1958-5772

Éditeur

ACEDLE

\section{Référence électronique}

Giovanni Agresti, « Le paradigme plurilingue pour une révolution humaniste », Recherches en didactique des langues et des cultures [En ligne], 18-1 | 2021, mis en ligne le 03 mai 2021, consulté le 03 août 2021. URL : http://journals.openedition.org/rdlc/8564; DOI : https://doi.org/10.4000/rdlc.8564

\section{Ce document a été généré automatiquement le 3 août 2021}

Recherches en didactique des langues et des cultures is licensed under a Creative Commons AttributionNonCommercial-NoDerivatives 4.0 International License 


\title{
Le paradigme plurilingue pour une révolution humaniste
}

\author{
Giovanni Agresti
}

\section{NOTE DE L'AUTEUR}

L'association italienne DORIF-Università, Centro di documentazione e di ricerca per la didattica della lingua francese nell'Università italiana et le réseau international POCLANDE, Populations, Cultures, Langues et Développement ont apporté leur soutien à ce texte.

1 À l'âge de la globalisation, la diversité linguistique est menacée. Ce constat n'est pas nouveau. Mais aujourd'hui nous commençons peut-être à prendre pleinement conscience de sa portée. La menace n'est guère anodine : vaste, pesant aussi bien sur les langues moins répandues que sur les langues nationales, elle est également profonde, mordant sur la vie des individus et conditionnant parfois lourdement les communautés.

2 L'hégémonie de la dimension économique et l'idolâtrie du marché global qui la couronne, triomphant des vieilles idéologies, représentent sans aucun doute des facteurs de risque majeurs. Sans visage, si ce n'est celui de l'argent, l'économie globale participe d'une microphysique du pouvoir qui colonise jusqu'à l'imaginaire. Ainsi, la crise de la diversité linguistique est, d'abord et surtout, une crise des pratiques culturelles traditionnelles et de leur transmission: sauf exception, on ne transmet aux nouvelles générations que ce que l'on imagine être économiquement rentable, bien entendu d'après le modèle dominant.

3 Ainsi, de toutes les formes que peut prendre aujourd'hui la protection de la diversité linguistique, il n'y a que la patrimonialisation des langues qui soit vraiment tolérée, voire récupérée par l'idéologie dominante. Ersatz compensatoire, réponse, a maxima, à une instance de réparation historique et, a minima, simple caution rhétorique, la patrimonialisation n'en rime pas moins avec «cristallisation» et, parfois, avec 
«folklorisation ». Elle se situe donc dans bien des cas aux antipodes d'une revitalisation et d'une re-fonctionnalisation réelles des langues. C'est juste du mieux que rien.

Par rapport au passé, il y a à présent un élément nouveau et décisif. Pour fragiliser la transmission intergénérationnelle des langues et des cultures, il n'est plus besoin de les stigmatiser, comme on a pu le faire, par exemple, au lendemain de la Révolution française, en s'en prenant aux « patois »- coupables présumés de plonger les esprits dans les ténèbres de l'ignorance. Aujourd'hui, rien que par sa force "gravitationnelle », c'est l'économie globale qui infirme et décrédibilise tout soutien à la diversité linguistique, dès lors que celle-ci se configure comme une barrière aux échanges des marchandises, à la vitesse de la communication et à la mobilité des individus.

5 La globalisation, telle qu'elle se présente aujourd'hui, n'est pas qu'une configuration économique planétaire: c'est une structure culturelle intégrale et incorporante. Tout se tient : à une économie globale correspondent une langue et une culture globales. Ainsi, par exemple, le dogme de la croissance économique illimitée, qui participe des fondements du marché global, ne peut se déployer que dans le cadre d'une société vorace de consommation, qui présuppose à son tour une communication simplifiée, incessante, purement instrumentale et un délit ou déni de conscience (ou d'attention) : notre Planète, ses ressources, ne sont pas illimitées, de même que notre vie. Le consumérisme entretient un mensonge, se basant sur une interaction qui n'en est pas une : c'est le régime de l'offre et de la demande. Et ce régime communicationnel entre fournisseurs de services et de produits et clients semble bien-former de plus en plus n'importe quelle interaction, jusqu'aux discours les plus intimes.

Il s'agit d'un échange biaisé, car il est caractérisé par une demande toujours croissante de biens, matériels et immatériels, dont la nécessité n'est très souvent qu'apparente. Ces besoins sont nourris d'une part par des fantasmes de bien-être, de liberté et de réussite individuels, et de l'autre par un sentiment d'insatisfaction permanente, de déclassement, par une fragilisation du sujet qui naît, aussi, de la pulvérisation de la mémoire collective et des réservoirs symboliques des communautés, abrités entre autres par les langues et les récits dont celles-ci sont porteuses. C'est en appauvrissant la dimension culturelle, mémorielle et spirituelle (au sens laïque du mot) du sujet, sa dimension linguistique et son autonomie intellectuelle, ses récits intérieurs, qu'on fait de celui-ci un besogneux consommateur d'émotions (stéréotypes), de conforts, de plaisirs, de distractions facilement accessibles. Dès lors, on peut compter sur son insatisfaction permanente et sa revendication du bonheur pour lui vendre sans cesse des appâts.

7 En polluant l'environnement qu'elle exploite pour s'alimenter à l'infini, en incorporant et bien-formant la société, la globalisation se doit d'assujettir et de polluer le langage et finit par contaminer les esprits. On a pu observer que les sociétés envahies par un langage stéréotypé (ou langue de bois) sont celles où les travailleurs sont le plus en perte de droits. Le système assujettit... le sujet, et ce à partir de ce que celui-ci a de plus irréductible : sa parole.

8 Comment tolérer, dès lors, la dérive monolingue ou oligolingue qui sévit aujourd'hui, qui prend l'aspect d'une dérive déshumanisante? Nul ne nie l'importance de quelques langues de grande communication internationale. Au contraire: ces instruments peuvent être, entre autres, le levier de changements multiples et rapides, par exemple dans le cadre de la technique et des mœurs, des styles de vie. Mais cette puissance de voix doit être équilibrée et maniée avec grande prudence, car en aucune manière elle ne devrait servir d'argument pour écraser le pluralisme linguistique et culturel. Loin de 
représenter une sorte de rédemption de l'humanité surgie de Babel, un aboutissement inéluctable, une marque sûre du progrès des civilisations occidentales, la dérive monolingue ou oligolingue doit être mise sévèrement en question, car elle risque de précipiter l'espèce vers sa ruine. La perte de diversité linguistique accompagne la perte de biodiversité, l'homologation et le conformisme culturels, la perte de créativité dans les sciences, avec des conséquences directes que l'on commence à chiffrer et des conséquences indirectes, à moyen et long terme, dont on peine à mesurer l'ampleur réelle.

Quoi qu'il en sera de la diversité linguistique, on peut déjà évaluer les effets néfastes de la globalisation économique. Au lieu de se poser en tant qu'agent démocratique de redistribution, la compétition globale finit par centraliser le pouvoir et provoque des déséquilibres redoutables aux niveaux économique, social, territorial (abandon des campagnes et des montagnes et concentration dans des villes toujours plus polluées et tentaculaires) et environnemental (impact dévastateur sur les milieux naturels). Force est de constater que la globalisation issue des politiques néolibérales n'a pas vraiment amélioré les conditions d'existence des sujets et des collectivités, creusant un fossé de plus en plus large et profond entre les très riches (de plus en plus riches et de moins en moins nombreux) et les pauvres (de plus en plus nombreux). Elle a mis à mal les classes moyennes, mis en danger l'équilibre écologique de notre maison commune et sanctionné des formes de dépendance économique voire de néocolonialisme de fait de peuples tout entiers via, par exemple, l'endettement, la privatisation de cultures traditionnelles à travers les brevets, le décalage et la soumission technologiques... Des conflits armés, des soulèvements sociaux réitérés, des formes de malaise ou d'aliénation semblent se multiplier un peu partout, sur fond de désertification culturelle.

10 La compétition globale, souvent érigée en gage de liberté, de démocratie et de progrès, est en réalité porteuse de conflits et semble miner le sentiment d'appartenance à plusieurs communautés à la fois : familiale, locale, régionale, nationale, continentale, mondiale. Cela ne peut que fragiliser les liens sociaux, délégitimer la diversité linguistique (ou cohabitation heureuse dans un même répertoire - individuel ou collectif - de langues locales, nationales, internationales etc.) et saper l'esprit de solidarité.

11 C'est pourquoi il convient de marquer une différence nette entre une globalisation marchande, agressive, centralisatrice, tendanciellement monolingue, et une mondialisation - et mobilisation - salutaire des esprits, une pensée-monde équilibrante et distributive, riche de la diversité des apports.

12 La pensée-monde est une pensée qui relève le défi de la complexité et accepte de se faire à la fois exigeante et humble. La globalisation marchande cultive en revanche le narcissisme et la paresse intellectuelle, en installant souvent un redoutable déterminisme : 1) on est malheureux parce que dépossédés de nous-mêmes faute de transmission/réception culturelle et mémorielle $>2$ ) on veut être heureux, on prétend en avoir le droit $>3$ ) on est infantilisés en raison de la désertification mémorielle et culturelle et on croit que le consumérisme nous guérira $>4$ ) d'ailleurs, tout peut s'acheter, il faut juste parler la même langue et adopter, partager un même discours. Le cercle se clôt. Le consensus fabrique la vérité. Langue et discours finissent par converger ou se confondre. Quid de la parole subjective? 
13 La réduction de la diversité linguistique et l'appauvrissement des capacités discursives et dialogales du sujet et des groupes semblent donc deux symptômes d'une même pathologie culturelle, deux effets d'un même processus de diminution du monde. C'est pourquoi, du point de vue théorique, il n'est plus suffisant de souhaiter protéger la diversité linguistique : il faut comprendre que c'est aussi par la diversité linguistique que passe notre avenir, individuel et civilisationnel. Le plurilinguisme individuel associé au multilinguisme sociétal devrait être pensé comme le soubassement d'une révolution humaniste plus que comme l'un de ses produits éventuels.

14 La résistance à la dérive monolingue s'organise. Elle prend parfois la forme du repli identitaire ou de la révolte solitaire, qui finalement finissent par reconduire le pouvoir dominant : le nationalisme, la xénophobie, le racisme sont hélas de mise aujourd'hui, et sont aussi le produit direct ou indirect de la globalisation marchande et des égoïsmes qu'elle produit et entretient. Mais cette résistance peut prendre également des formes plus articulées, fécondes et exigeantes. Celles-ci passent principalement par la mise en valeur aussi bien de la dimension sociale des échanges que de l'épaisseur de la parole humaine et de la mémoire. Contre toute fermeture, individuelle ou communautariste, l'ouverture à l'altérité peut se réaliser moyennant la prise en compte des langues et des récits de ceux qui ont été davantage exclus jusque-là : les gardiens de mémoire, les passeurs de mémoire, les relais intergénérationnels, les sans-voix, les sujets minoritaires. Des femmes, des hommes, riches en expérience de vie, au plus près de la vie et du contact humain, habités par le sentiment de la schise et de la limite. Mais on ne peut véritablement se dire que par notre langue maternelle.

Il y a là un constat majeur: au lieu de les protéger nous, ce sont nos langues maternelles qui nous protègent, car elles s'enracinent au plus profond de notre être et permettent, contre toute tentation de repli, de "faire communauté » et de nous projeter dans le monde de la manière la plus authentique qui soit. La désaliénation individuelle et collective passe par là. Voilà pourquoi la révolution nécessaire aujourd'hui doit être à la fois sociale, humaniste et langagière. Dans cette perspective, loin d'être une finalité ou de se configurer comme un tournant épistémologique, les extraordinaires innovations technologiques qui marquent notre époque ne peuvent jouer que le rôle d'outils, d'instruments accélérateurs. Les technologies qu'il faut développer seront de plus en plus celles de l'esprit, les ressources intérieures. Le développement du sujet ne devrait pas se mesurer à l'aune de sa " capacité de risque ", comme le prétendrait la "culture" financière, ou de sa capacité à gagner la compétition, à écraser l'autre. Le sujet "développé » ne saurait être non plus un consommateur : il doit être vu, pensé, ressenti comme un écrin de mémoire et de vie, un bijoutier de l'expérience, un chef cuisinier du partage. Il doit être conscient des richesses qui l'habitent. Il deviendra par conséquent moins besogneux d'objets matériels, de prothèses technologiques, et plus souverain de sa parole: sans pour autant céder à la facilité d'une parole publique déréglée, bon marché et faussement démocratique; sans pour autant reconduire une parole autrui mille fois copiée et collée ; sans pour autant se laisser emporter par des effets de mode. Cette parole libérée du sujet - profonde, réflexive, ciselée, créatrice - sera la synthèse des paroles plurielles qui l'ont traversé, en présence (ses propres interactions), en absence (les interactions indirectes), en latence (la mémoire de langue que la communauté à laquelle il appartient recèle en son sein). 
C'est dans la perspective de conquête ou reconquête de cette parole à la fois créatrice et enracinée que le paradigme plurilingue doit être mis au cœur d'un projet novateur de société. Ce paradigme met l'accent sur le sujet, confronté à plusieurs altérités, qui participent de sa construction culturelle, linguistique, émotionnelle et mémorielle. Un sujet plurilingue est un sujet qui, le plus souvent, a peiné à le devenir, et qui est particulièrement sensible et conscient de la nature de la langue, du discours et, donc, des enjeux de la communication. Un sujet plurilingue saura dire différemment les mêmes objets, saura les mettre en perspective, et aura sans doute quelques outils supplémentaires pour échapper aux représentations stéréotypes du monde et à ses violences symboliques.

Nous aimerions dire que le sujet plurilingue est le vrai citoyen de l'Europe et de la Méditerranée, d'aujourd'hui comme de demain. Inlassable passeur de frontières, il lui incombe de tisser, de coudre des relations entre les nombreuses pièces d'une mosaïque linguistique et culturelle qui, de par sa profondeur historique, n'a sans doute pas d'équivalent dans le monde. Au lieu de ressentir de plus en plus de distance à l'égard des institutions continentales, comme c'est malheureusement le cas aujourd'hui, le vrai citoyen de l'Europe et de la Méditerranée doit se sentir investi de leur construction : la richesse mémorielle et culturelle de cette région, on ne devrait jamais l'oublier, est le résultat d'une interaction plurimillénaire de ses habitants qui a débouché sur des synthèses qui sont des monuments de civilisation. Penser que ce formidable creuset culturel a besoin, aujourd'hui, d'une configuration monolingue et monoculturelle, sous prétexte d'«internationalisation » et de compétition globale, est le leurre ultime. La révolution sociale, langagière et humaniste est, tout comme au début de l'humanisme, une question de rencontres, d'échanges, d'apprentissages et de déplacements à l'intérieur de ce Continent et de cette Mer qui est la nôtre et qui est le carrefour, ou plutôt la charnière, de trois continents.

Nous proposons là juste les prémisses idéologiques d'une intervention sur la façon d'entendre et de valoriser la diversité linguistique et culturelle et dans la manière d'agir pour faire remonter les ressources d'ordre prioritairement social que celle-ci recèle. Ce programme est assurément fait de multiples actions, qui touchent les mondes de la recherche et de la formation autant que l'engagement politique et civique. Bien entendu, il n'y a pas de solutions clés en main. Mais l'alerte est lancée, le débat est ouvert et ne se refermera pas demain.

\section{AUTEUR}

\section{GIOVANNI AGRESTI}

Université Bordeaux Montaigne, France

Giovanni Agresti est professeur en Sciences du langage à l'Université Bordeaux Montaigne. Il a défini la « linguistique du développement social ». Président du réseau scientifique international POCLANDE (Populations, cultures, langues et développement), il a organisé à compter de 2007 des conférences internationales annuelles à l'Université de Teramo (Italie), les Journées des Droits 
Linguistiques, devenues en 2015 le $1^{\text {er }}$ Congrès mondial des droits linguistiques. Membre du Comité scientifique de l'Académie Internationale de Droit Linguistique de Montréal et professeur honoraire de l'International University of Fundamental Studies de Moscou.

Courriel : giagresti[at]yahoo.it 